Artículo

\title{
Evaluación continua y cooperativa: el caso del Bachillerato en Informática Empresarial, un primer acercamiento
}

Continuous and cooperative assessment: the case of the Bachelor of Business Computing career, a first approach

Denis González Herrera *

dennis.gonzalezherrera@ucr.ac.cr

ISSN 1996-1642, Editorial Universidad Don Bosco, año 10, No.17, enero-junio de 2016, pp. 61-71

Recibido: 4 de mayo 2015. Aprobado: 18 de diciembre de 2015.

Resumen

El presente estudio aborda la evaluación desde las estrategias formativas $y$ cooperativas en el ambiente universitario. Se destaca aquí la aplicación de la evaluación sumativa, diagnóstica, autoevaluativa y coevaluativa, así como la importancia de una cultura de evaluación en el contexto universitario. Se exponen diversas estrategias de evaluación que permitan al docente universitario considerar alternativas sobre la evaluación sumativa al brindar una base teórica para evidenciar y fomentar el uso de otras estrategias evaluativas adecuadas en los ambientes universitarios. Se finaliza, abordando la contextualización de la experiencia adquirida en la Universidad de Costa Rica, Sede de Occidente.

Palabras clave: Estrategias evaluativas, evaluación formativa, evaluación cooperativa, Universidad de Costa Rica.
Abstract:

This paper approaches evaluation viewed from formative and cooperative strategies in the university context. It is highlighted how diagnostic, auto evaluation and peer evaluation, as well as innovative evaluation culture can be implemented in such context. Besides, this paper overviews a diversity of assessment strategies that enables the university teacher to overpass summative evaluation and provides a theoretical basis to elicit and promote the use of other assessment strategies. To finish, there is an analysis of the evaluation experience at the University of Costa Rica, western campus.

Keywords: Assessment strategies, formative evaluation; collaborative evaluation; University of Costa Rica

* Profesor del Departamento de Ciencias Naturales, Sede Occidente, UCR, Costa Rica. 


\section{Introducción}

Uno de los elementos más importantes en los procesos de enseñanzaaprendizaje que se desarrollan en un ambiente universitario, tiene que ver con la forma de evaluar el aprendizaje del estudiantado (Salas, 1991). Como docentes críticos, en constante búsqueda del mejoramiento continua y la obtención de la excelencia académica (Universitario, 1974) dentro de los procesos educativos universitarios, se cuestiona ¿Cuál podría ser la mejor estrategia evaluativa que garantice calidad y excelencia de la educación dentro de nuestro contexto y que además considere las particularidades del área del saber a desarrollar y junto con el tipo de discentes que queremos formar?

La presente investigación está centrada en el estudio de las estrategias evaluativas formativa y cooperativa al considerar que representan una alternativa sobre la estrategia actual mayormente adoptada por la Universidad de Costa Rica: evaluación sumativa. Es posible caracterizar a esta evaluación según el uso de pruebas estandarizadas, a la utilización de instrumentos como tareas, pruebas cortas, comprobación de lecturas, trabajos de investigación, cuestionarios y exámenes entre otros. Estas actividades buscan medir el logro alcanzado por el estudiantado, el cual se ve reflejado con la suma de sus porcentajes al final del semestre (Valverde, 2011 ). El mismo autor evidencia algunos de los problemas de este tipo de evaluación, como por ejemplo, la evaluación es muy selectiva y discriminatoria, únicamente se establece por el criterio subjetivo del docente y es utilizada como un medio de control unidireccional entre otras falencias.

El objetivo del estudio es brindar una base teórica para evidenciar y fomentar el uso de otras estrategias evaluativas de impacto y adecuadas a un ambiente universitario que puedan fortalecer, complementar o sustituir las estrategias evaluativas actuales. Es decir, desarrollar un marco conceptual con la intención de exponer diversas estrategias de evaluación que permitan al docente universitario considerar diversas alternativas sobre la evaluación. Esto tomando como base que existe una gran variedad de estrategias evaluativas entre las que se mencionan la formativa o continua, la sumativa, diagnóstica, autoevaluativa, coevaluación y cooperativa, entre otras.

Para evidenciar el impacto práctico de este estudio se expondrán los resultados de incorporar la estrategia evaluativa cooperativa en conjunto con la evaluación sumativa, en un ambiente universitario: curso de Sistemas Operativos de la carrera de Bachillerato en Informática Empresarial, perteneciente al segundo año del plan de estudio, con una duración de 16 semanas.

Evaluación continua y cooperativa: el caso del Bachillerato en Informática Empresarial, un primer

2. acercamiento

\section{Marco conceptual}

\section{La evaluación educativa}

La evaluación puede ser concebida según las necesidades, propósitos u objetivos institucionales. Los diferentes autores la conceptualizan e interpretan de maneras muy distintas según sus usos, fines o intenciones, siguiendo principios o normas particulares. Indudablemente todos con la idea de lograr la mejor 
evaluación y la mayor calidad. La evaluación presenta actividades (calificar, medir, corregir, clasificar, certificar, examinar), que desempeñan un papel funcional e instrumental, pero de estas actividades no se aprende; al trascender estas actividades identificamos a la evaluación en el ámbito educativo como aprendizaje en el sentido que por ella adquirimos conocimiento (Méndez, 2001).

Para Jorba y Sanmartí (1993), la evaluación como regulación involucra tres etapas: recogida de información, análisis de la información y juicio sobre el resultado y la toma de decisiones de acuerdo con el juicio. De esta manera la evaluación de los aprendizajes desde su función pedagógica, regula el proceso de enseñanzaaprendizaje reconociendo los cambios que se han de introducir paulatinamente durante este proceso, con la intención de lograr que el estudiantado aprenda de manera significativa. Dicha función es de carácter pedagógico o formativo, pues aporta información útil para la adaptación de las actividades de enseñanzaaprendizaje a las necesidades del alumnado y de este modo mejorar la calidad de la enseñanza en general (Jorba y Sanmartí, 1993).

Evaluar es conocer, con el fin fundamental de asegurar el progreso formativo de quienes aprenden y junto con ellos de quienes enseñan, es decir, la evaluación se convierte en actividad continua de conocimiento (Cruz y Quiñones, 2012).

Como se ha expuesto, el concepto de evaluación es más amplio que un simple sistema de calificaciones tradicionales. La evaluación requiere de la actuación del profesorado y del alumnado. Según Gómez (2010), la evaluación educativa es una reflexión crítica sobre los componentes y los intercambios en el proceso didáctico, con el fin de determinar cuáles están siendo o han sido sus resultados y poder tomar las decisiones más adecuadas para la positiva consecución de los objetivos educativos. De esta forma indica que la evaluación requiere tiempo, trabajo colaborativo y aceptación al cambio en razón del mejoramiento continuo.

De igual forma, Grau (2005 citado por Gómez, 2010) indica que como proceso de aprendizaje, la evaluación educativa trata de constatar los cambios que se han producido en el alumnado, la eficacia de los métodos y de los recursos empleados, la adecuación de los títulos, programas o asignaturas y, en general, todos los factores que puedan incidir en la calidad educativa, para así, poder tomar las decisiones oportunas que permitan reconducir (si fuera necesario) el proceso de enseñanza-aprendizaje hacia los fines que se pretendían. De modo que, ambos aspectos, el de" juicio" y el de "toma de decisiones" intervienen en la evaluación educativa, aunque adquieren mayor o menor preponderancia según los casos (Rosales, 2014).

La evaluación, como una estrategia, es útil y necesaria para el mejoramiento de la calidad de la educación (Mora, 2004). La evaluación vista como un proceso debe consensuar múltiples actores según sus diversos intereses, valores y puntos de vistas. Como actores podemos mencionar al estudiantado, al profesorado y a la misma universidad. Las diversas estrategias utilizadas deben garantizar la 
participación de cada uno de ellos con la intención de lograr los objetivos de enseñanza-aprendizaje, en busca del mejoramiento continuo.

Tal como lo simplifican Bordas y Cabrera (2001), al decir que el énfasis en las nuevas tendencias de evaluación es la participación de las personas, las estrategias evaluativas deben buscar una evaluación formadora, multicultural, participativa y consensuada, centrada en el aprendizaje.

\section{Tipos de evaluación}

Evaluación formativa o continua la retroalimentación al estudiante es un elemento clave para que la evaluación pueda considerarse formativa. De igual modo, deben abordar la evaluación como parte del proceso de enseñanza y, sobre todo, como parte del proceso de aprendizaje del estudiante (Jiménez, Sáez y Gómez, 2014).

Para que la retroalimentación resulte verdaderamente formativa para el estudiante se debe comunicar la información necesaria con el fin de modificar el pensamiento del estudiante o su comportamiento con el propósito de mejorar el aprendizaje (Shute, citado por Jiménez y Flores, 2014).

Para Rosales (2014) es la que se realiza durante el desarrollo del proceso de enseñanza-aprendizaje para localizar las deficiencias cuando aún se está en posibilidad de remediarlas, esto es, introducir sobre la marcha rectificaciones a que hubiere lugar en el proyecto educativo y tomar las decisiones pertinentes, adecuadas para optimizar el proceso de logro del éxito por el alumnado.

Según Jorba y Sanmartí (1993), este tipo de evaluación responde a una concepción de la enseñanza que considera que aprender es un largo proceso a través del cual el alumnado va reestructurando su conocimiento a partir de las actividades que lleva a cabo. En resumen persigue los siguientes objetivos: la regulación pedagógica, la gestión de los errores y la consolidación de los éxitos.

Evaluación sumativa Tiene por objeto establecer balances fiables de los resultados obtenidos al final de un proceso de enseñanza-aprendizaje; tiene esencialmente, una función social de asegurar que las características de los estudiantes respondan a las exigencias del sistema (Jorba y Sanmartí, 1993).

Cruz y Quiñones (2012) exponen que la calificación no tiene sentido por sí misma, es necesario contextualizarla dentro del proceso, darle un significado educativo; es el resultado de un proceso que el estudiante ha vivido, y debe basarse en información confiable y válida sobre su desempeño.

Evaluación continua y cooperativa: el caso del Bachillerato en Informática Empresarial, un primer

64. acercamiento
La evaluación sumativa engloba todos aquellos exámenes, ejercicios o pruebas que se realizan, única y exclusivamente, con objeto de comprobar si el alumnado conoce o no el contenido de una disciplina (Olmos, 2008). Su necesidad radica en la responsabilidad del sistema educativo de certificar si el estudiantado está o no capacitado para la obtención de un determinado título. 
Coevaluación Cuando se evalúa un mismo trabajo, o actividad por varios sujetos a la vez se está realizando una coevaluación, evaluación entre "colegas", donde la responsabilidad está claramente compartida (Olmos, 2008). Topping (1998, citado por Jiménez 2006, p.3) define la coevaluación como la "disposición en la cual los individuos consideran la cantidad, nivel, valor, calidad o éxito de los productos o resultados del aprendizaje de compañeros de igual estatus".

Orsmond, Merry y Reiling (citado por Jiménez 2006) encontraron que los estudiantes sintieron que adoptaron actitudes más críticas, que trabajaron de una manera más estructurada y que este tipo de evaluación les hizo aprender y pensar más. En palabras de Millis y Cottell (citado por Jiménez 2006, p.5) "los estudiantes pueden ser capaces de engañar a su profesor o profesora, pero raramente podrían esconderse de sus propios compañeros".

Tal y como se indica anteriormente, la coevaluación se refiere a la evaluación realizada mutuamente entre compañeros, la cual brinda mecanismos e instrumentos que permiten a un grupo de alumnos evaluarse entre ellos; de tal forma que les permitan dar retroalimentación y pautas durante el desarrollo de una evaluación comparativa entre iguales. La participación del alumnado juega, por tanto, un papel crucial en el ajuste individual de la calificación conseguida por el grupo cooperativo (Jiménez, 2006).

En cuanto al aprendizaje en grupos cooperativos, la coevaluación quizá sea la única manera satisfactoria de evaluar cómo trabaja un conjunto de estudiantes en un proyecto de grupo (Brown, Bull y Pendlebury, citado por Jiménez, 2006).

Autoevaluación Algunas de las definiciones que se han realizado sobre el término expresan; según Mora (2004) consiste en un proceso de análisis y reflexión introspectivo y prospectivo acerca del propio quehacer educativo, necesario para mejorar el servicio que brinda una institución educativa.

Para García y Cuello (2009) es un tipo de evaluación ligada con el aprendizaje autónomo y con el aprendizaje a lo largo de la vida, ya que es una evaluación que realiza el propio estudiante y que le permite comprobar su propio nivel de aprendizaje y, en su caso, reorientarlo.

Este tipo de evaluación es caracterizado por García y Cuello (2009) en un escenario donde el estudiantado se convierte en el protagonista de su proceso de aprendizaje, aumentando su motivación, su compromiso y su responsabilidad. Además el estudiantado recibe un retorno de su proceso, comprueba qué conocimientos y competencias ha asimilado y desarrollado correctamente y cuáles no, por cual motivo y cómo puede mejorarlas. En síntesis la autoevaluación es un proceso de análisis y reflexión que acerca al propio quehacer educativo tanto interior como exteriormente.

Evaluación cooperativa McConnell (citado por Jiménez 2006) indica que es un efecto al aprendizaje cooperativo y constituye una verdadera colaboración 
entre alumnado y profesorado ya que las dos partes trabajan en el objetivo común de proporcionar una evaluación consensuada del conocimiento del estudiantado.

Es importante destacar que para los docentes, la evaluación cooperativa supone ceder parte de la responsabilidad del proceso de evaluación en los estudiantes (Jiménez, 2006).

En un proyecto cooperativo es necesario definir los niveles de participación de todos los involucrados, así como trabajar en la coordinación de las actividades de evaluación. Es importante que el estudiantado se sienta parte del proceso y de la toma de decisiones.

Las habilidades para autoevaluarse, para realizar evaluaciones entre iguales o para participar en evaluaciones cooperativas son importantes en el desarrollo del aprendizaje a lo largo de la vida del estudiante y en el desarrollo de la autonomía de los individuos (Sambell, McDowell y Brown, citado por Rosales, 2014).

\section{Cultura de evaluación}

La evaluación está orientada por una cultura institucional (leyes, reglamentos, decretos y circulares) y por la cultura evaluativa; ésta se construye a través del conjunto de valores internalizados por los participantes del proceso formativo: el estudiantado, el profesorado, administrativos, padres y empleadores, acerca de la forma de concebir y practicar la evaluación en un determinado proceso educativo (Duque, citado por Mora, 2004).

De igual manera, Bolseguí y Fuguet (2006) indican que puede estar conformado por un conjunto de reglas, hábitos, rutinas, creencias explícitas y tácitas, estructuras, símbolos, premisas básicas, valores, estrategias, historia, métodos y técnicas, que comparte una comunidad en relación con los procesos de evaluación que allí se han desarrollado y se desarrollan. El concepto cultura de evaluación, apunta al anclaje de los procesos de evaluación en las instituciones del sector de educación superior, a la necesidad de instaurar la costumbre de evaluar y de atribuir mayor importancia a la evaluación, como proceso asociado al cambio y a la transformación universitaria.

Generar la cultura de la evaluación en los centros, supone asumir una nueva forma de trabajar en los mismos, orientada a la mejora continua de la calidad y una nueva forma de evaluarlas utilizando como herramienta la autoevaluación (García, 2005).

Evaluación continua y cooperativa: el caso del Bachillerato en Informática Empresarial, un primer

66. acercamiento

\section{El contexto de la evaluación}

Actualmente, en la actividad educativa universitaria se prioriza el uso de la evaluación sumativa, exámenes finales que ayuden a los docentes a poner "notas" que el propio sistema educativo exige para acreditar si los alumnos están o no preparados para continuar sus estudios o incorporarse a la vida activa, 
laboral (Olmos, 2008). El autor también señala que la evaluación formativa no es la que prima en la educación universitaria.

Bordas y Cabrera (2001) presenta algunas características de la evaluación tradicional frente a la evaluación participativa. Entre los aspectos tradicionales se menciona: el poder en el profesor, la función de enseñanza del profesorado se legitima según su carácter evaluador, se limita al sistema de evaluación, evaluación centrada en los resultados y se evidencia la pasividad del alumnado; además, las evaluaciones son obra del profesorado sin la participación del estudiantado. En relación a la evaluación participativa se expone: el poder emana del consenso, el estudiantado como evaluador aprende a conocer y a dirigir su proceso de aprendizaje, se enfatiza en la cooperación y el estudiantado es activo y cooperativo en su evaluación.

Al ser la carrera en Informática Empresarial (IE) (Sede de Occidente) parte integral de la Universidad de Costa Rica, comparte por igual sus características en materia de evaluaciones. Como se ha mencionado, IE realiza mayoritariamente una evaluación de carácter sumativa, respondiendo al aspecto social de la evaluación que garantiza el cumplimiento mínimo para la obtención de un título universitario. De esta manera la participación del estudiantado en materia de evaluación es pasiva resumiendo su actuación a la realización de pruebas, por lo general escritas y obteniendo una calificación al final de un semestre: un valor numérico. Esto se puede evidenciar en el Universitario (1974), en él se cita: La evaluación del estudiante en una asignatura se calificará mediante números en una escala de 0 a 10. También se menciona que la calificación del alumno es el resultado de la evaluación durante el período lectivo y de la nota del examen final del curso. A pesar que el Universitario (1974) representa una guía y los detalles de las evaluaciones son adjuntadas y pueden ser modificadas de forma consensuada en los programas de los diferentes cursos por el profesorado y el estudiantado, la evaluación siempre debe representar una calificación numérica.

Además el Universitario (2001) indica que la calificación es exclusiva del personal docente asignado para tal efecto, dejando sin pronunciamiento la participación del estudiantado en los procesos de evaluación. La carrera en IE conserva una función de evaluación certificadora, limitando su función generadora de los aprendizajes. Al investigar sobre la evaluación cooperativa y formativa en el ambiente IE, se busca avanzar y motivar un cambio institucional y universitario con el fin de lograr una mejora y una innovación en la cultura de los procesos de evaluación actuales. Con esto se pretende ayuda a generar ideas nuevas que permitan una evaluación para el aprendizaje continuo, consensuado y con una cultura de evaluación institucional e individual que facilite la búsqueda de la calidad y la obtención de la excelencia académica.

\section{Las TIC y la evaluación}

La innovación en las aplicaciones de tecnologías web y el actual crecimiento de las TIC se convierten en plataformas idóneas para potenciar y apoyar los procesos de enseñanza-aprendizaje. Pueden promover una mejor relación 
entre profesorado y estudiantado al desarrollar dinámicas de cooperación entre el alumnado favoreciendo un aprendizaje más colaborativo, Chao (2014). Las TIC fomentan la participación activa del estudiantado en los procesos de enseñanza-aprendizaje y facilitan la retroalimentación. Además permiten aumentar el tiempo, el contacto y la participación de cualquier participante en los procesos educativos. Estas características posibilitan la creación de estructuras de comunicación colaborativas y de acceso omnipresente, dentro de los espacios de enseñanza y fuera de ellos. El potencial mostrado en el uso de las TIC dentro de la educación, ha elevado el interés por los educadores, ya que aporta ventajas de acceso, flexibilidad de tiempo y espacio para el desarrollo de actividades. De igual forma Soto, Senra, y Neira (2009) indican que las TIC permiten la creación de nuevos entornos comunicativos y expresivos que abren la posibilidad de desarrollar nuevas experiencias formativas. También brindan soporte para gestionar de manera rápida y ágil, diversas actividades desgastantes y repetitivas para el docente, como la recolección de tareas, la continua calificación y corrección de ejercicios. Se convierte en un medio ideal para suministrar retroalimentación rápida y de fácil acceso durante el proceso evaluativo.

\section{Metodología}

El estudio realizado a lo largo del semestre consistió en la utilización de al menos una herramienta de TIC que permitiera el trabajo colaborativo durante las lecciones semanales y facilitara la evaluación y corrección de los aportes que el estudiantado realizaba durante las clases presenciales. Cabe destacar que se mantuvo la estrategia de evaluación sumativa tradicional basada en: quizzes (exámenes cortos), tareas y exámenes durante todo el desarrollo del ciclo lectivo y la estrategia de evaluación colaborativa se utilizó como complemento, ya que no es posible sustituir por completo la estrategia evaluativa sumativa definida por la Universidad; por esta razón se optó por incorporar la estrategia de evaluación colaborativa únicamente en la calificación de los laboratorios del curso.

\section{Población}

Participaron 15 de las 18 personas matriculadas en el curso de sistemas operativos de la carrera de Bachillerato en Informática Empresarial, Sede de Occidente, perteneciente al segundo año del plan de estudio. De los cuales 4 corresponden a mujeres, 11 a hombres, con un rango de edad de 18 a 20 años. Todos los participantes habían matriculado el curso por primera vez.

\section{El instrumento}

La aplicación del instrumento se desarrolló en la semana 14 del curso, momento en el cual se habían realizado todas las actividades y evaluaciones

Evaluación continua $y$ cooperativa: el caso del Bachillerato en Informática Empresarial, un primer

68. acercamiento con excepción del examen final. El instrumento consistía en un cuestionario de preguntas cerradas y abiertas solicitando información sobre los objetivos, metodología didáctica, contenidos y las actividades de evaluación realizadas durante las lecciones previas; finalizando con las opiniones sobre mejoras y la valoración del desempeño del profesorado durante las 14 semanas. 


\section{El proceso y los recursos}

Se utilizó la herramienta de mediación virtual Moodle como plataforma especializada en contenidos de aprendizaje al igual como la define Gorospe (2005). También permite la gestión y comunicación entre el profesorado y el estudiantado; todo el material didáctico y el acceso a las diferentes herramientas de TIC colaborativas eran administradas en Moodle como puerta de acceso. Entre las aplicaciones de TIC utilizadas figuran los documentos de Google doc definido por Caivano (citado por Fonseca, Medellín, y Vásquez, 2014), específicamente los documentos de texto y de hoja de cálculo. También se utilizaron herramientas tipo pizarra virtual: padlet y lino; las cuales permiten gestionar aportes de tipo multimedia: texto, audio, vídeo, imágenes e hipertexto permitiendo la interacción entre profesorado y estudiantado. Éstas pueden generar un tipo de mapa conceptual que puede ser evaluado como parte de una actividad. Durante el desarrollo de un determinado laboratorio el estudiantado iba desarrollando la actividad según los objetivos y los contenidos propuestos por el profesorado. Los aportes del estudiantado son compartidos inmediatamente con todos los participantes de la clase, profesorado y estudiantado. Posteriormente pueden ser evaluados por cualquiera de sus compañeros o por el profesorado.

Estas tecnologías colaborativas permiten el empoderamiento del estudiantado sobre sus procesos de enseñanza-aprendizaje; facilitando su propia evaluación y permitiendo la retroalimentación inmediata mediante los aportes de sus compañeros. Es probable que está utilización conduzca a un uso ventajoso de la tecnología (TIC) que beneficia el aprendizaje del estudiantado.

\section{Resultados}

Con relación a las preguntas orientadas a valorar el curso, un 53\% respondió que el curso fue muy útil y un $47 \%$ lo considera útil. El $53 \%$ del estudiantado también indica estar muy satisfecho con el cumplimiento de los objetivos, mientras que el $43 \%$ se encuentra satisfecho. Además el $67 \%$ indica que la propuesta programática del curso se cumplió de manera satisfactoria y un 33\% de muy satisfactoria. Al preguntar sobre el cumplimiento de expectativas del curso un $60 \%$ se muestra satisfecho y un $40 \%$ muy satisfecho.

Con respecto a las preguntas enfocadas a las actividades, metodología, técnicas didácticas y evaluativas se muestra que el $67 \%$ expresa estar muy satisfecho en relación a la metodología y las técnicas didácticas, el 33\% indica sentirse satisfecho. El enfoque del material utilizado durante las clases en relación con los contenidos de la propuesta programática se valoró como apropiado por parte del 73\% de los encuestados, mientras que el 27\% lo evaluó como muy apropiado. Finalmente al consultar si las actividades y trabajos de evaluación colaborativos desarrollados durante el curso favorecen la reflexión, la creatividad y el aprendizaje el $87 \%$ indica estar totalmente de acuerdo y un $13 \%$ de acuerdo. 
En relación a las preguntas abiertas solicitando comentarios o sugerencias se destacan la siguientes respuestas: "Se utilizó técnicas de evaluación nuevas, interesantes y buenas", "Seguir con actividades que involucren más la puesta en práctica de los conocimientos por parte del estudiante", "Se abarca la clase dando un mutuo acuerdo y provocando mayor interés por la materia" y "las variaciones en las actividades de trabajo dieron dinamismo al curso", entre otros comentarios que destacan los aspectos innovador, dinámico, entretenido y amigable del curso.

\section{Conclusión}

Realizar un proceso alternativo en materia de evaluación de los aprendizaje en la Sede de Occidente, desde una perspectiva formativa y colaborativa; es una valiosa oportunidad para generar realimentación que fortalezca el quehacer académico e institucional, facilitando el desarrollo de procesos de enseñanzaaprendizaje más efectivos.

Es necesario destacar la excesiva importancia otorgada a las calificaciones en el ambiente universitario durante la evaluación sumativa la cual deja de lado la obtención de los objetivos de aprendizaje, olvidando aspectos esenciales en el proceso didáctico, como la forma en que el estudiantado aprende. A pesar que la evaluación sumativa puede proporcionar retroalimentación que ayude a la toma de decisiones, dicha información no es inmediata por lo que no se puede realizar rectificación sobre errores o dificultades en el momento en que el estudiantado contemple; lo que puede generar repercusiones negativas en el desarrollo del proceso educativo.

Es indudable que los estudiantes son responsable en gran parte de su propio aprendizaje, por esta razón también deberían ser ellos responsables de su evaluación y de sus calificaciones, integrando la evaluación como parte de su aprendizaje. Nadie mejor que el sujeto que aprende para conocer lo que realmente sabe (Méndez, 2001).

Además del mecanismo y de los procedimientos de evaluación tradicionales (evaluación sumativa), y al margen de otros tipos de evaluación como la formativa, autoevaluación, coevaluación, se debe contemplar la posibilidades de otras estrategias que responsabilicen al estudiantado en su propia evaluación y por lo tanto, en su propio aprendizaje.

Es importante indicar que el modelo teórico aquí presentado sólo representa una explicación provisional e invita a continuar con el proceso de reflexión

Evaluación continua y cooperativa: el caso del Bachillerato en Informática Empresarial, un primer

70. acercamiento

\section{Referencias}

Bolseguí, M. y Fuguet, A. (2006). Cultura de evaluación: una aproximación conceptual. Investigación y Postgrado, 21(1), 77-98. 
Bordas, M. I. y Cabrera, F. (2001). Estrategias de evaluación de los aprendizajes centrados en el proceso. Revista española de pedagogía, 218, 25-48.

Chao, K. W. C. (2014). Estrategias didácticas mediadas con tic en un curso de expresión oral francesa. Revista Electrónica Actualidades Investigativas en Educación, $14(2), 1-30$.

Cruz Núñez, F. y Quiñones Urquijo, A. (2012). Importancia de la evaluación y autoevaluación en el rendimiento académico. Zona Próxima (16).

Fonseca Chiu, L. B., Medellín Serna, L. A., y Vásquez Padilla, J. L. (2014). El uso de herramientas de la web 2.0 como estrategias didácticas en el proceso de enseñanza-aprendizaje de jóvenes universitarios. PAAKAT, (7).

García, A. M. y Cuello, R. O. (2009). Interacción entre la evaluación continua y la autoevaluación formativa: la potenciación del aprendizaje autónomo. Revista de Docencia Universitaria (4 8).

García, B. (2005). La cultura de la evaluación como proceso de mejora de un centro educativo.

Gómez Lucas, C. (2010). La evaluación, un proceso de cambio para el aprendizaje.

Gorospe, J. M. C. (2005). La integración de plataformas de e-learning en la docencia universitaria: Enseñanza, aprendizaje e investigación con Moodle en la formación inicial del profesorado. Revista Latinoamericana de Tecnología EducativaRelatec, 4(1), 37-48.

Jiménez, E. G., y Flores, A. M. (2014). La retroalimentación automática en las pruebas objetivas a través de Internet.

Jiménez, E. G., Sáez, M. S. I., y Gómez, G. R. (2014). Análisis de las innovaciones en la evaluación en la Educación Superior: La perspectiva de profesores y estudiantes.

Jiménez Valverde, G. (2006). Obtención de notas individuales a partir de una nota de grupo mediante una evaluación cooperativa. Revista lberoamericana de Educación (OEI) 38(5).

Jorba, J., y Sanmartí, N. (1993). La función pedagógica de la evaluación. Aula de innovación educativa, 20, 20-30.

Méndez, J. M. Á. (2001). Evaluar para conocer, examinar para excluir. Morata.

Mora, V. (2004). La Evaluación Educativa: Concepto, Períodos y Modelos. Revista Actualidades Investigativas en Educación, 4(2), 1-28.

Olmos Miguelañez, S. (2008). Evaluación formativa y sumativa de estudiantes universitarios: aplicación de las tecnologías a la evaluación educativa.

Rosales, M. (2014). Proceso evaluativo: evaluación sumativa, evaluación formativa y Assesment su impacto en la educación actual. Congreso lberoamericano de Ciencia, Tecnología, Innovación y Educación, Argentina 2014.

Salas Perea, R. (1991). El papel de la evaluación en el proceso de enseñanza-aprendizaja. Educ. med. super, 5(1), 3-17.

Soto, C. A.; Senra, A. I. y Neira, M. D. C. (2009). Ventajas del uso de las TICs en el proceso de enseñanza-aprendizaje desde la óptica de los docentes universitarios españoles. Revista electrónica de tecnología educativa, (29), 5.

Universitario, C. (1974). Estatuto Orgánico de la Universidad de Costa Rica. Recuperado de http://www.cu.ucr.ac.cr/normativ/estatuto_organico.pdf.

Universitario, C. (2001). Reglamento de Régimen Académico estudiantil.

Valverde, A. A. y Vargas, M. B. (2011). La evaluación integral y alternativa en la Sede de Occidente a partir de procesos de investigación-acción. InterSedes, 10 (18). 